\title{
Stress-strain state of the short eccentrically compressed reinforced concrete columns with nonlinear creep
}

\author{
Anton Chepurnenko ${ }^{1 *}$, Ludmila Dubovitskaya ${ }^{2}$, Vladimir Vanja ${ }^{3}$, Damian Israel Tenemaza \\ Ortega $^{1}$, and Andrey Kuznetsov ${ }^{4}$ \\ ${ }^{1}$ Don State Technical University, 344002, Rostov-on-Don, Russia \\ ${ }^{2}$ Russian State University of Tourism and Service, 141221, Moscow region, Cherkizovo, Russian \\ Federation \\ ${ }^{3}$ Kuban State Agrarian University named after I.T. Trubulin, 350044, Krasnodar, Russia \\ ${ }^{4}$ Belgorod National Research University, 308015, Belgorod, Russia
}

\begin{abstract}
The article presents the derivation of the resolving equations for calculating short eccentrically compressed reinforced concrete elements taking into account the creep of concrete. The case of symmetric reinforcement is considered. A viscoelastoplastic model of hereditary aging by A.G. Tamrazyan is used. Comparison of solutions based on linear and nonlinear creep theory is performed.
\end{abstract}

\section{Introduction}

We consider a short reinforced concrete rod, which is acted upon by an eccentric force (Fig. $1)$.
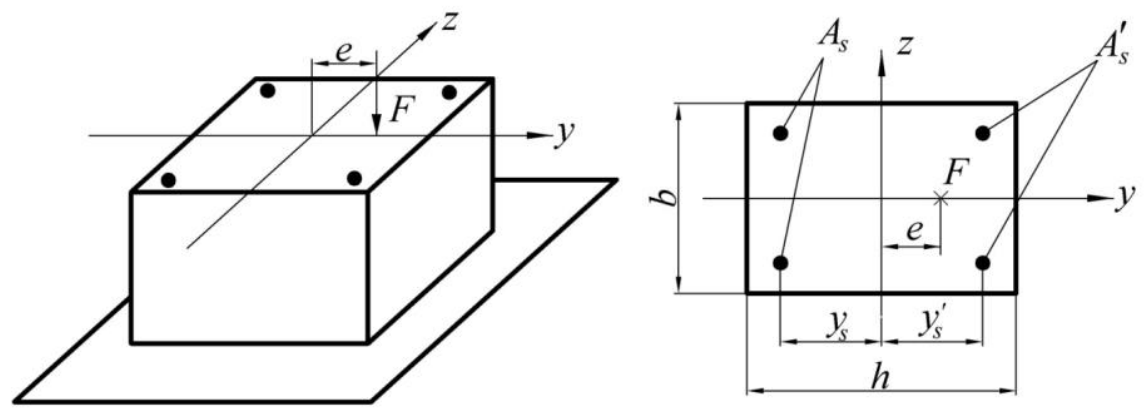

Fig. 1. Calculation scheme

When deriving the resolving equations, we will use the visco-elasto-plastic model of hereditary aging [1]. In accordance with this model, the relationship between deformations and stresses is as follows:

\footnotetext{
* Corresponding author: anton chepurnenk@mail.ru
} 


$$
\varepsilon(t)=\frac{f[\sigma(t)]}{E(t)}-\int_{\tau_{0}}^{t} f[\sigma(\tau)] \frac{\partial C(t, \tau)}{\partial \tau} d \tau
$$

where $f[\sigma(t)]$ - some stress function, $C(t, \tau)$ - creep measure.

The first term on the right-hand side of (1) is the instantaneous deformation, which is the sum of the elastic and plastic components. The second term is the creep deformation that develops in time.

\section{Methods}

Equation (1) can be written in a slightly different way:

$$
\varepsilon(t)=\frac{\sigma(t)}{\bar{E}(\sigma, t)}+\varepsilon^{*}
$$

where $\bar{E}(\sigma, t)-$ secant modulus of elasticity, $\varepsilon^{*}=-\int_{\tau_{0}}^{t} f[\sigma(\tau)] \frac{\partial C(t, \tau)}{\partial \tau} d \tau-$ creep deformation.

To solve the problem, the method of successive approximations will be used, which in the literature is often called the method of variable parameters of elasticity [2]. The essence of this method is that a physically nonlinear problem is reduced to the sequential solution of elastic problems for an inhomogeneous body. Therefore, to begin with, it is necessary to obtain the governing equations for an eccentrically compressed rod, in which the modulus of elasticity of concrete varies with height, i.e. $E_{b}=f(y)$.

Equilibrium equations will be written in the form:

$$
\begin{gathered}
F=-b \int_{-h / 2}^{h / 2} \sigma_{b} d y-\sigma_{S} A_{S}-\sigma_{S}^{\prime} A_{S}^{\prime} \\
M=F e=b \int_{-h / 2}^{h / 2} \sigma_{b} y d y-\sigma_{S} A_{S} y_{S}+\sigma_{S}^{\prime} A_{S}^{\prime} y_{S}^{\prime}
\end{gathered}
$$

where $\sigma_{b}$-stresses in concrete, $\sigma_{S}$ and $\sigma_{S}^{\prime}$ - reinforcement stresses.

The total deformation of concrete, according to the hypothesis of flat sections, will be written in the form:

$$
\varepsilon_{b}=\varepsilon_{0}-y \cdot æ
$$

where $\varepsilon_{0}$ - axial deformation, $æ=\frac{d^{2} v}{d x^{2}}-$ rod curvature.

Equating the deformations of the reinforcement to the deformations of concrete, we get:

$$
\begin{aligned}
& \varepsilon_{S}=\varepsilon_{0}+y_{S} \cdot æ, \varepsilon_{S}^{\prime}=\varepsilon_{0}-y_{S}^{\prime} \cdot æ
\end{aligned}
$$

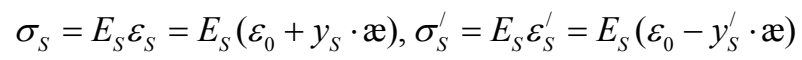

Let us express from (2) the stresses in concrete:

$$
\sigma_{b}=\overline{E_{b}}(y)\left(\varepsilon_{b}-\varepsilon_{b}^{*}\right)=\overline{E_{b}}(y)\left(\varepsilon_{0}-y \cdot \mathfrak{x}-\varepsilon_{b}^{*}\right)
$$

Substituting (7) and (6) in (4), for the case of symmetric reinforcement ( $y_{S}=y_{S}^{\prime}, A_{S}=A_{S}^{\prime}$ ) we obtain: 


$$
M=-E I_{r e d} æ-b \int_{-h / 2}^{h / 2} \overline{E_{b}}(y) \varepsilon^{*} y d y+b \varepsilon_{0} \int_{-h / 2}^{h / 2} \overline{E_{b}}(y) y d y,
$$

where $E I_{\text {red }}=E_{S}\left(A_{S} y_{S}^{2}+A_{S}^{\prime}\left(y_{S}^{\prime}\right)^{2}\right)+b \int_{-h / 2}^{h / 2} \overline{E_{b}}(y) y^{2} d y$ - reduced bending stiffness of the cross-section.

We find the value $\varepsilon_{0}$ by substituting (7) into (3):

$$
\varepsilon_{0}=\frac{1}{E A_{r e d}}\left(-F+b \int_{-h / 2}^{h / 2} \overline{E_{b}}(y) \varepsilon^{*} d y+b æ \int_{-h / 2}^{h / 2} \overline{E_{b}}(y) y d y\right),
$$

where $E A_{\text {red }}=b \int_{-h / 2}^{h / 2} \overline{E_{b}}(y) d y+E_{S} A_{S, \text { total }}$ - reduced stiffness of the cross section at central compression.

We substitute (9) in (8):

$$
\begin{gathered}
\mathfrak{x}\left[-E I_{\text {red }}+\frac{b^{2}}{E A_{\text {red }}}\left(\int_{-h / 2}^{h / 2} \overline{E_{b}}(y) y d y\right)^{2}\right]=M+b \int_{-h / 2}^{h / 2} \overline{E_{b}}(y) \varepsilon^{*} y d y+ \\
+\frac{F b}{E A_{\text {red }}} \int_{-h / 2}^{h / 2} \overline{E_{b}}(y) y d y-\frac{b^{2}}{E A_{\text {red }}} \int_{-h / 2}^{h / 2} \overline{E_{b}}(y) \varepsilon^{*} d y \cdot \int_{-h / 2}^{h / 2} \overline{E_{b}}(y) y d y .
\end{gathered}
$$

From expression (10), knowing at a given moment of time the distribution $\overline{E_{b}}(y)$ and deformation of creep, it is possible to find the curvature of the rod. Further, the value $\mathfrak{x}$ is substituted in (9) and then stresses in reinforcement and concrete are determined by formulas (6) and (7).

Let us turn to the definition of creep deformations. According to [1], the creep measure can be presented in the most generalized form as follows:

$$
C(t, \tau)=\sum_{i=1}^{m} \theta_{i}(t) e^{-\alpha_{i} \tau}
$$

Then the creep deformation can be represented as the sum of the components:

$$
\varepsilon^{*}=\sum_{i=1}^{m} \varepsilon_{i}^{*}, \quad \varepsilon_{i}^{*}=\alpha_{i} \theta_{i}(t) \int_{\tau_{0}}^{t} f[\sigma(\tau)] e^{-\alpha_{i} \tau} d \tau
$$

Let's differentiate (12) by time:

$$
\begin{aligned}
\frac{\partial \varepsilon_{i}^{*}}{\partial t}= & \alpha_{i}\left(\frac{\partial \theta_{i}(t)}{\partial t} \int_{\tau_{0}}^{t} f[\sigma(\tau)] e^{-\alpha_{i} \tau} d \tau+\theta_{i}(t) \cdot \frac{\partial}{\partial t}\left(\int_{\tau_{0}}^{t} f[\sigma(\tau)] e^{-\alpha_{i} \tau} d \tau\right)\right)= \\
& =\alpha_{i}\left(\frac{\partial \theta_{i}(t)}{\partial t} \int_{\tau_{0}}^{t} f[\sigma(\tau)] e^{-\alpha_{i} \tau} d \tau+\theta_{i}(t) \cdot f[\sigma(t)] e^{-\alpha_{i} t}\right) .
\end{aligned}
$$

We exclude the integral from expression (13). To do this, we express it from (12):

$$
\begin{aligned}
& \int_{\tau_{0}}^{t} f[\sigma(\tau)] e^{-\alpha_{i} \tau} d \tau=\frac{\varepsilon_{i}^{*}}{\alpha_{i} \theta_{i}(t)} \text { and substitute in }(13): \\
& \qquad \frac{\partial \varepsilon_{i}^{*}}{\partial t}=\frac{\partial \theta_{i}(t)}{\partial t} \frac{\varepsilon_{i}^{*}}{\theta_{i}(t)}+\alpha_{i} \theta_{i}(t) \cdot f[\sigma(t)] e^{-\alpha_{i} t} .
\end{aligned}
$$


The resulting expression is very convenient for practical calculations.

Consider the methodology for solving the problem. The time interval at which creep is considered is divided into $n$ steps $\Delta t$, and the cross-section is divided into $m$ parts $\Delta y$ along the height. At the first stage, we solve the problem for $t=\tau_{0}$ and $\varepsilon^{*}=0$. As the first approximation, we assume that $\bar{E}_{b 1}=$ const . We determine the stresses and use them to find the secant module $\bar{E}_{b}\left(\sigma_{1}\right)$ at each point. In the second approximation, we replace $\bar{E}_{b 1}$ with $\bar{E}_{b 2}=\left(\bar{E}_{b 1}+\bar{E}_{b}\left(\sigma_{1}\right)\right) / 2$, etc.

Let us denote by $\left\{\sigma_{i}\right\}=\left\{\begin{array}{llll}\sigma_{1 i} & \sigma_{2 i} & \ldots & \sigma_{m i}\end{array}\right\}$ the vector whose coordinates are the values of stresses at each point in the i-th approximation. Starting from the 2 nd approximation, we find the vector $\{\Delta \sigma\}=\left\{\sigma_{i}\right\}-\left\{\sigma_{i-1}\right\}$ and its norm $\sqrt{\{\Delta \sigma\} \cdot\{\Delta \sigma\}^{T}}$, as well as the vector $\left\{\sigma_{i}\right\}$ norm.

The criterion for exiting the loop will be the following condition:

$$
\sqrt{\frac{\{\Delta \sigma\} \cdot\{\Delta \sigma\}^{T}}{\left\{\sigma_{i}\right\} \cdot\left\{\sigma_{i}\right\}^{T}}} \cdot 100 \%<0.1 \% .
$$

Having determined the stresses at $t=\tau_{0}$, we find, using formula (14), the growth rates of the creep strain components. The values $\varepsilon_{i}^{*}$ at the moment of time $t+\Delta t$ are found using the linear approximation:

$$
\varepsilon_{i, t+\Delta t}^{*}=\varepsilon_{i, t}^{*}+\frac{\partial \varepsilon_{i}^{*}}{\partial t} \Delta t
$$

Such a technique for determining creep deformations is used in works [3-7]. Further, at the next moment in time, we also use the method of successive approximations.

In [1] it is recommended to use the creep measure in the form:

$$
C(t, \tau)=C \frac{e^{\alpha t}-e^{\alpha \tau}}{e^{\alpha t}-1}+B\left(e^{-\gamma \tau}-e^{-\gamma t}\right) .
$$

Expression (17) can also be written in the form (11):

$$
\begin{gathered}
C(t, \tau)=\sum_{i=1}^{5} \theta_{i}(t) e^{-\alpha_{i} \tau} ; \theta_{1}(t)=C ; \alpha_{1}=0 ; \\
\theta_{2}(t)=-\frac{C}{e^{\alpha t}-1} ; \alpha_{2}=-\alpha ; \theta_{3}(t)=\frac{C}{e^{\alpha t}-1} ; \alpha_{3}=0 ; \\
\theta_{4}(t)=B ; \alpha_{4}=\gamma ; \theta_{5}(t)=B e^{-\gamma t} ; \alpha_{5}=0 .
\end{gathered}
$$

However, with this creep measure, the creep deformation can be represented as a sum of not five, but only two components [8-10].

\section{Results and Discussion}

The problem was solved with the following initial data: the initial modulus of elasticity of concrete at $\tau_{0}=28$ days : $E_{0}\left(\tau_{0}\right)=3 \cdot 10^{4} \mathrm{MPa}$, prismatic strength $R=20 \mathrm{MPa}$, crosssectional dimensions $b=20 \mathrm{~cm}, h=40 \mathrm{~cm}$, modulus of elasticity of reinforcement $E_{S}=2 \cdot 10^{5} \mathrm{MPa}$, design compressive strength of reinforcement $R_{s c}=400 \mathrm{MPa}$, 
reinforcement ratio $\mu=\frac{A_{S, \text { total }}}{A}=0.03, F=1200 \mathrm{kN}, e=4 \mathrm{~cm}, y_{S}=y_{S}^{\prime}=15 \mathrm{~cm}$. Rheological parameters $C=3.77 \cdot 10^{-5} \mathrm{MPa}^{-1}, \alpha=0.032$ days $^{-1}, B=5.68 \cdot 10^{-5} \mathrm{MPa}^{-1}$, $\gamma=0.062$ days $^{-1}$.

The dependence of the secant module on stress was taken in the form [1]:

$$
\bar{E}(\sigma, t)=E_{0}(t)\left[1+\beta \sigma^{n-1}\right]^{-1},
$$

where $\beta=\frac{66-R}{(R-6) R^{2}}, R$ is the prismatic strength in MPa.

Expression (19) is equivalent to the stress function $f(\sigma)=\sigma\left[1+\beta \sigma^{n-1}\right]$. The coefficient $n$ was taken equal to 2. Compressive stresses in expressions for $\bar{E}(\sigma, t)$ and $f(\sigma)$ should be substituted with a "+" sign.

The dependence of the initial modulus of elasticity on time was taken in the form:

$$
E_{0}(t)=E_{0}\left(\tau_{0}\right)\left[b_{1}+\left(1-b_{1}\right) e^{-b_{2}\left(t-\tau_{0}\right)}\right]
$$

where $b_{1}=1.282, b_{2}=0.019$.

Fig. 2 shows the graph of the change in concrete stresses over time. It can be seen that at the initial moment of time the stress distribution along the height is nonlinear. Over time, the stresses in concrete at the most compressed face decrease in absolute value, and practically do not change at the less compressed face. Fig. 3 shows the change in time of relative stresses $\sigma_{S} / R_{S C}$ and $\sigma_{S}^{\prime} / R_{S C}$. The dashed line corresponds to the solution according to the linear theory (at $f(\sigma)=\sigma$ ). Over the time, an increase in stresses in the reinforcement occurs due to the transfer of the part of the perceived forces from the concrete. According to the nonlinear theory, the stresses in the reinforcement turn out to be greater than along the linear one.

Fig. 4 is the distribution of stresses in concrete along the section height at $t=\tau_{0}=28$ days and at $t=100$ days. The dashed line is the linear theory solution. The "+" sign in fig. 4 corresponds to compression. Both at the beginning and at the end of the creep process, the results differ significantly. The linear theory gives overestimated values of stresses at the most compressed face. 


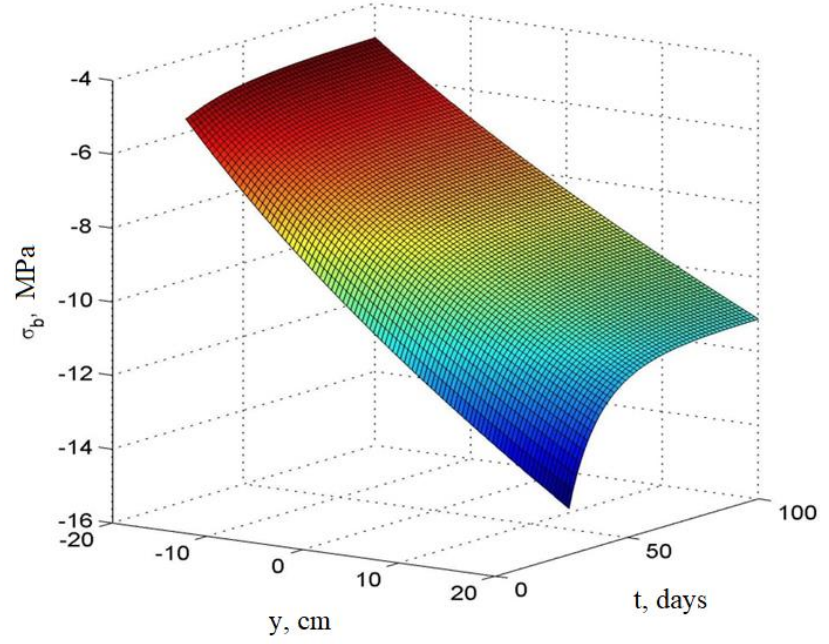

Fig. 2. Change in concrete stresses over time

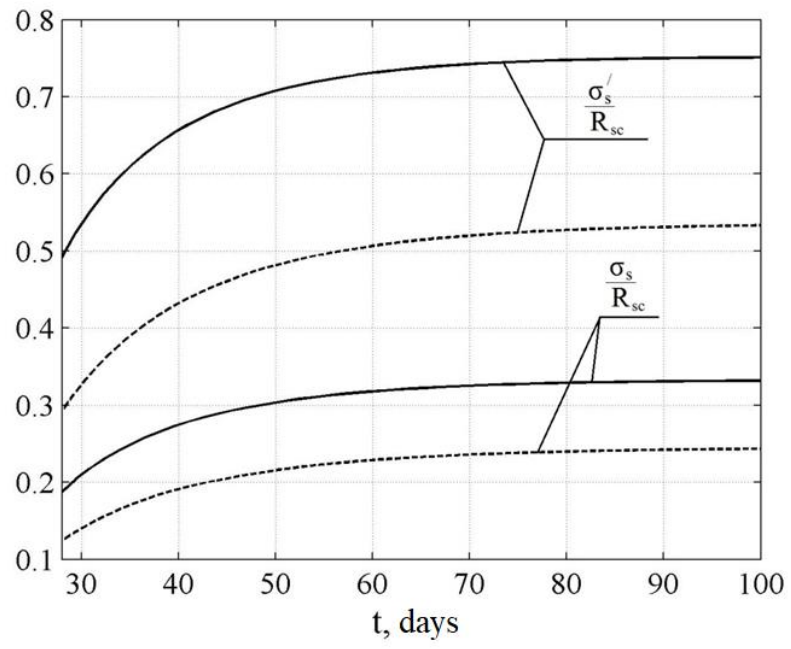

Fig. 3. Change in the relative stresses in reinforcement over time

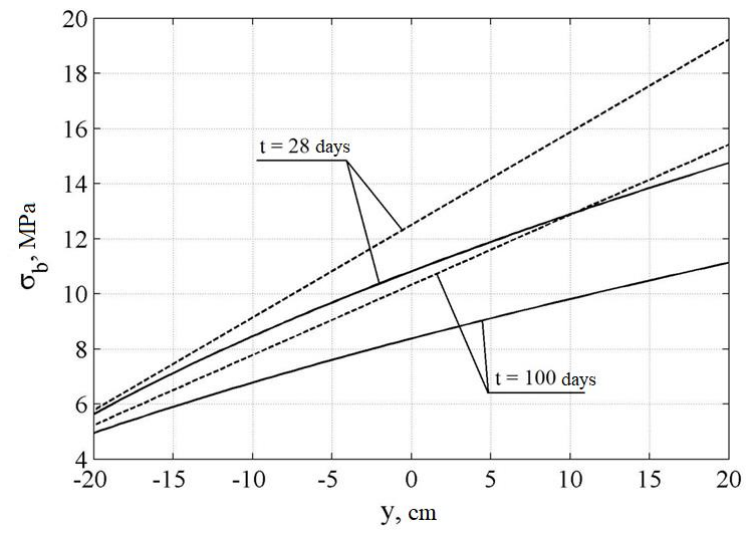

Fig. 4. Distribution of stresses along the height of the section 


\section{Conclusion}

Resolving equations are obtained and the calculation method for eccentrically compressed reinforced concrete short columns is developed on the basis of the viscoelastoplastic model of hereditary aging of concrete. These equations are suitable for arbitrary relationships between stresses and instantaneous deformations.

The theoretical study of the stress-strain state of the eccentrically compressed short reinforced concrete column with nonlinear creep was carried out using the analytical expression of A.G. Tamrazyan for short-term loading diagrams. The comparison is presented between the results obtained using nonlinear and linear theory. It was found that, according to the nonlinear theory, the redistribution of stresses between concrete and reinforcement is more significant than along the linear one.

\section{References}

1. A.G. Tamrazyan, S.G. Yesayan, Concrete creep mechanics: monograph (Moscow, MGSU, 2012)

2. V.I. Andreev, Some problems and methods of mechanics of inhomogeneous bodies: monograph (Moscow, Publishing house ASV, 2002)

3. V. Chepurnenko, B. Yazyev, X. Song, MATEC Web of Conferences 129, 05009 (2017)

4. S. B. Yazyev et al., MATEC Web of Conferences 129, 05010 (2017)

5. V. Chepurnenko, B. Yazyev, I. Dubovitskaya, E3S Web of Conferences 164, 02003 (2020)

6. S.V. Litvinov et al., International Polymer Science and Technology 42, 23-25 (2015)

7. S. Litvinov, L. Trush, S. Yazyev, Materials Science Forum 935, 121-126 (2018)

8. L.R. Mailyan, A.S. Chepurnenko, A. Ivanov, Procedia Engineering 165, 1853-1857 (2016)

9. A.S. Chepurnenko, L.R. Mailyan, B.M. Yazyev, A. Ivanov, MATEC Web of Conferences 106, 04010 (2017)

10. A.S. Chepurnenko, N.V. Neumerzhitskaya, M.S. Turko, Advances in Intelligent Systems and Computing 692, 808-818 (2017) 